\title{
Changements climatiques et déterminants d'adoption de la fumure organique dans la région semi-aride de Kibwezi au Kenya
}

\author{
Abou SALE ${ }^{1}$, Denis Pompidou FOLEFACK ${ }^{1 *}$, Gilbert Obati OBWOYERE ${ }^{2}$, \\ Nakhone LENAH WATI ${ }^{2}$, Wirnkar Venasius LENDZEMO ${ }^{1}$ et Anselme WAKPONOU ${ }^{3}$ \\ ${ }^{1}$ Institut de Recherche Agricole pour le Développement (IRAD), B.P. 33 Maroua, Cameroun. \\ ${ }^{2}$ Crop Management, Research and Training Centre (CMRT), University of Njoro, Kenya. \\ ${ }^{3}$ Université de Ngaoundéré, B.P. 455 Ngaoundéré, Cameroun. \\ "Auteur correspondant, E-mail: dfolefack@yahoo.fr
}

\section{RESUME}

Cet article analyse les facteurs socio-économiques qui influencent l'utilisation de la fumure organique face à la baisse de la fertilité des sols dans un contexte de changements climatiques dans la région semi-aride de Kibwezi. Un modèle Logit est estimé pour isoler les variables affectant la décision d'utiliser la fumure organique afin de corriger cette baisse de fertilité des sols, à partir des données collectées auprès des producteurs. Les résultats montrent une corrélation entre les variables socio-économiques et l'utilisation de la fumure organique face à la baisse de la fertilité des sols. Ainsi, on constate que les variables socioéconomiques telles que le statut matrimonial, les connaissances techniques locales, l'accès aux ressources, les relations sociales dans le village d'une part, et le niveau d'éducation des chefs de ménages d'autre part, ont respectivement une influence significative sur la probabilité d'utilisation de la fumure organique aux seuils de $5 \%$ et de $10 \%$. Les autres facteurs potentiels n'ont aucun effet significatif sur la probabilité d'utiliser la fumure organique pour corriger la baisse de fertilité des sols dans la région. Enfin, pour accroître le taux d'adoption de cette stratégie, une sensibilisation plus accrue des producteurs sur l'importance de la fumure organique doit être faite.

(C) 2014 International Formulae Group. All rights reserved.

Mots clés : Changements climatiques, Fumure organique, Modèle Logit, Zone semi-aride, Kenya.

\section{INTRODUCTION}

Les changements climatiques et la dégradation des terres constituent des menaces pour la survie et les moyens de subsistance de millions de personnes en Afrique subsaharienne (Sultan, 2011). La forte dépendance des économies et des populations rurales vis-àvis de l'agriculture non irriguée, la pauvreté et l'insécurité alimentaire, ainsi que le faible développement des capacités institutionnelles et des infrastructures sont tels que l'adaptation à la variabilité naturelle du climat est un défi toujours d'actualité (Sultan, 2011 ; Berg et al., 2012).

La fertilité des sols est en baisse dans de nombreuses régions d'Afrique subsaharienne. Les changements climatiques devraient 
occasionner des événements météorologiques plus extrêmes comme des inondations, des sécheresses et du temps plus imprévisible (Folefack et al., 2012). Ces changements ne feront vraisemblablement qu'accentuer les problèmes de fertilité des sols qui diminue pour diverses raisons. Certaines pratiques agricoles courantes, dont le brûlage des résidus de culture et l'abandon des sols à nu sans protection contre le soleil et le vent sont évoquées (Hansen, 2002 ; Nacro et al., 2010). L'utilisation excessive ou insuffisante d'engrais et les rotations de cultures inadéquates entraînent également une diminution de la fertilité des sols (Zombré, 2003 ; PNUD, 2004). $\mathrm{Au}$ cours de ces dernières années, le nombre d'incidents climatiques violents dans certaines sous-régions d'Afrique et le nombre de personnes victimes des sécheresses et des inondations ont enregistré une forte augmentation (Kieken, 2007). En effet, de nombreux modèles climatiques prévoient que le changement climatique aura des répercussions négatives sur la production agricole et la sécurité alimentaire de vastes zones d'Afrique sub-saharienne. La dégradation des terres peut aggraver ces changements climatiques, mais elle peut également être exacerbée par ces derniers. Une autre incidence immédiate plus notoire de la dégradation des sols est que les paysans sont entraînés dans un cercle vicieux de pauvreté (Oram, 1989 ; Hansen et al., 2006 ; Challinor et al., 2007 ; FAO 2008). Dans la région de Kibwezi qui fait partie de la région semi-aride du Kenya, les sécheresses engendrées par les changements climatiques constituent des phénomènes cycliques qui font partie de la vie normale des populations (Salé, 2010). Néanmoins, il a été constaté dans cette région que depuis les années 70 , les sécheresses sont de plus en plus récurrentes, avec des conséquences plus catastrophiques (MOA, 2009). Les populations pauvres comme celles de la région de Kibwezi, sont particulièrement vulnérables aux effets des changements climatiques en raison de leur plus grande dépendance vis-à-vis de l'agriculture pluviale et de leur plus faible capacité d'adaptation (Salé, 2010).

Cependant, il existe des pratiques traditionnelles et modernes qui peuvent contribuer à améliorer la fertilité des sols et aider les agriculteurs à rendre leurs fermes plus résilientes et résistantes au climat changeant. Dans la région de Kibwezi, les principales stratégies d'adaptation relatives aux effets des changements climatiques sur les sols sont les terrasses et la fumure organique (Salé, 2010).

Plusieurs études ont été réalisées en Afrique sur les adaptations des producteurs aux changements climatiques, mais très peu ont été faites sur les déterminants de ces stratégies, pour montrer le rôle des facteurs socio-économiques sur l'adoption (Folefack et al., 2012), d'où cette étude pour contribuer à combler cette lacune. L'enjeu pour la recherche est de concevoir et d'utiliser avec les acteurs des outils de modélisation pouvant aider le producteur à concevoir des modes de gestion de la fumure organique afin d'améliorer durablement les performances techniques et la fertilité des sols de l'exploitation.

L'objectif visé par cette étude est d'identifier les variables socio-économiques qui influencent l'utilisation de la fumure organique face à la baisse de la fertilité des sols dans un contexte de changements climatiques dans la région semi-aride Kibwezi au Kenya. 


\section{MATERIEL ET METHODES}

\section{Zone d'étude et collecte des données}

Cet article est rédigé à partir des résultats de l'étude menée par Salé Abou en 2010 sur les changements climatiques et les stratégies des agriculteurs dans la région semiaride de Kibwezi au Kenya dans le cadre d'African Climate Change Fellowship Program à l'Université Egerton de Njoro au Kenya. Le département de Kibwezi, situé au sud-est du Kenya a été choisi, car, il fait partie des régions semi-arides (250-500 $\mathrm{mm})$ de l'Afrique subsaharienne où les populations sont sérieusement affectées par les effets des changements climatiques (MOA, 2009). Les agro-éleveurs particulièrement sont plus touchés à cause de l'impact notoire des ces changements sur les ressources telles que les cultures, les sols, les animaux et l'eau. Comme conséquences, il y a fontes de semis, baisse des rendements agricoles, famine, maladies humaines et animales, braderie d'animaux, longs parcours pour de l'eau. Néanmoins, pour des besoins d'adaptation, ces populations ont développé des techniques très diverses (Salé, 2010).

Un questionnaire structuré a été administré à 186 chefs de ménages choisis au hasard, mais seulement 111 ont été utilisés en raison des imprécisions relevées dans la collecte des données par les enquêteurs. Des analyses ont permis de constater que sur notre échantillon, 43 utilisent la fumure organique face à la baisse de la fertilité suite aux changements climatiques, soit $39 \%$ et 68 ne l'utilisent pas, soit $41 \%$. Les données ont été collectées au moyen des enquêtes à travers un questionnaire au cours de la campagne agricole 2009/2010.

Les agriculteurs ont fourni des informations actuelles et rétrospectives sur leurs exploitations, notamment: les caractéristiques socioéconomiques des familles (âge, sexe, statut matrimonial, etc.), les facteurs institutionnels (l'accès à l'information, l'accès aux services de vulgarisation, l'accès aux formations, l'accès aux services d'encadrement, l'accès au crédit, etc.), la situation agro-écologique, les facteurs climatiques (baisse des précipitations, hausse des températures).

\section{Modèle empirique et conceptuel}

Dans cette étude, nous faisons l'hypothèse que les variables socioéconomiques influencent l'utilisation de la fumure organique face à la baisse de la fertilité des sols suite aux changements climatiques. L'étude des déterminants du choix s'appuiera sur un modèle de choix discret avec comme objectif d'estimer conjointement la probabilité de chaque alternative par rapport à une alternative de référence. Le modèle économétrique requis est alors un modèle de type Logit ou Probit selon la loi de distribution retenue pour les termes d'erreur (Maddala, 1985 ; Greene, 1991; Long, 1997). Les modèles Logit polytomiques non ordonnés sont moins intensifs en calculs mais requièrent la non violation de l'hypothèse de l'indépendance des alternatives non pertinentes (Madalla, 1985; Cramer, 1991). Les modèles Probit polytomiques non ordonnés quand à eux sont plus intensifs en calculs et n'imposent pas de restrictions particulières. Dans ce travail, nous utiliserons le modèle Logit, qui facilite la manipulation des résultats (Hurlin, 2003).

Le principe fondamental du modèle Logit est basé sur la probabilité pour un individu de choisir ou non une stratégie ou le produit qui lui est proposé (Maddala, 1983, cité par Adésina et al., 2000 ; Varian, 2006).

Les paramètres de ce modèle Logit sont estimés par la méthode du maximum de vraisemblance (Nkamleu et Kielland, 2006; Nkamleu et Coulibaly, 2000; Nkamleu et 
Adesina, 2000 ; Chow, 1983). Le choix de l'exploitant dépend des opportunités et est, par conséquent, aléatoire et ne saurait faire l'objet d'une régression linéaire, mais d'une régression multiple qui peut être du type exponentiel (Greene, 1991 ; Nkamleu et Coulibaly, 2000). La décision d'adoption d'une stratégie peut être théoriquement conçue comme suit et indiquée dans les équations.

En effet, deux propriétés font l'intérêt de la fonction de répartition logistique dans la modélisation des choix discrets. Il s'agit notamment de son intervalle qui se réduit à $[0$, 1] et de la possibilité d'être linéarisée par une transformation logarithmique. Dans ce modèle, on définit une variable $\mathrm{y}^{*}$ comme suit : $\mathrm{y}_{\mathrm{i}}{ }^{*}=\alpha+\mathrm{x}_{\mathrm{i}} \beta+\varepsilon_{\mathrm{i}}, \quad$ (1) Où :

$\mathrm{Y}_{\mathrm{i}}^{*}$ représente le bénéfice ou l'intérêt retiré par l'exploitant de son engagement dans le choix d'une stratégie d'adaptation aux changements climatiques dans son exploitation; Xi est une variable qui peut influencer la pratique ;

$\beta$ les coefficients associés aux différentes variables du modèle et

ci l'erreur associée à la variable.

La variable $\mathrm{Y}^{*}{ }_{\mathrm{i}}$ n'étant pas observable, il est nécessaire de générer une variable observable exprimant le choix d'une stratégie par l'exploitant :

- $\mathrm{y}=1$, si le producteur utilise la fumure organique face à la baisse de la fertilité des sols et

- $\quad \mathrm{y}=0$, si le producteur n'utilise pas la fumure organique face à la baisse de la fertilité des sols.

Selon Hurlin (2003), la régression du modèle Logit caractérisant le choix par un échantillon d'exploitant est spécifié comme suit :

$$
p_{\mathrm{i}}=\mathrm{E}\left(\mathrm{y}_{\mathrm{i}}\right)=\mathrm{F}\left(\alpha+\mathrm{X}_{\mathrm{i}} \beta\right)=\frac{1}{\left[1+e^{-\left(\alpha+\mathrm{X}_{i} \beta\right)}\right]}
$$

où l'indice « $\mathrm{i}$ » indique la $\mathrm{i}^{\mathrm{ème}}$ observation dans l'échantillon, Pi est la probabilité qu'un individu fasse un choix donné, $\mathrm{y}_{\mathrm{i}}$ est la base du logarithme népérien, $\mathrm{x}_{\mathrm{i}}$ est un vecteur des variables exogènes, $\alpha$ est une constante et $\beta_{\mathrm{i}}$ sont des coefficients associés à chaque variable explicative $\mathrm{Xi}$ à estimer.

Les paramètres de ce modèle Logit sont estimés par la méthode du maximum de vraisemblance (Nkamleu et Kielland, 2006 ; Nkamleu et Coulibaly, 2000 ; Nkamleu et Adesina, 2000 ; Chow, 1983).

Il convient de noter que les coefficients estimés n'indiquent pas directement l'effet du changement des variables explicatives correspondantes sur la probabilité (p) de l'occurrence des résultats. Un coefficient positif signifie que la probabilité augmente avec l'accroissement de la variable indépendante correspondante (Neupane et al., 2002). Les coefficients $\alpha$ et $\beta$ dans la régression logistique sont estimés en utilisant la méthode du maximum de vraisemblance. Ainsi, le Logit est basé sur la notion de maximum de vraisemblance dont la fonction est :

$$
\prod_{i=0}^{n} \prod_{j=0}^{k}\left[\operatorname{Exp}\left(\beta_{j} x_{i j}\right) / \operatorname{Exp}\left(\beta_{j} x_{i j}\right)\right]^{N i j}
$$

Où $\mathrm{N}$ représente le nombre d'observations en dehors de celles pour lesquelles $\mathrm{Y}$ prend la valeur $\mathrm{j}$.

Toutefois, la fiabilité des paramètres estimés (convergence et normalité asymptotique) par cette méthode repose sur le caractère aléatoire et indépendant des variables explicatives utilisées; ce qui suppose que leurs valeurs sont déterministes et donc bornées. Pour résoudre le problème de multi colinéarité qui rendrait les résultats pas très fiables et inefficaces, nous avons procédé à une Analyse des Correspondances Multiples (ACM). Ainsi, 
dans nos estimations, les variables retenues sont celles qui offrent un plus grand pouvoir d'explication. Le Tableau 1 présente les variables explicatives utilisées dans le modèle.

L'utilisation de la fumure pour s'adapter à la baisse de la fertilité des sols face aux changements climatiques (USMARASO) : Cette variable est la variable dépendante qui est influencée par des facteurs socio-économiques. Cette stratégie constitue l'une des plus utilisées par les agriculteurs pour s'adapter aux changements climatiques.

L'âge du producteur (AGE) : cette variable mesurée en nombre d'années, constitue aussi un proxy de l'expérience dans les cultures agricoles. Les producteurs moins expérimentés sont jeunes. Les jeunes producteurs sont enclins à prendre plus de risque que les plus âgés.

Le sexe des enquêtés (GENDER) : les hommes ont plus accès à l'information et aux intrants comparés aux femmes. Ils auraient une plus grande probabilité d'adopter les technologies grâce à ces facteurs qui facilitent l'adoption.

Le statut matrimonial des enquêtés (MARSTAT) : cette variable est un proxy déterminant pour l'adoption des technologies agricoles. Le statut matrimonial détermine les besoins et les dépenses du producteur, et conditionne ses décisions d'améliorer sa productivité agricole. Les agriculteurs mariés ont plus de disponibilité de main d'œuvre et par conséquent, peuvent adopter plus facilement une stratégie d'adaptation.

Le niveau d'éducation (LEVEDUC) : cette variable est un proxy de la capacité managériale du producteur.

Elle indique aussi la capacité du producteur à disséquer les informations relatives à l'environnement économique et aux changements climatiques. Les producteurs éduqués ont de meilleures aptitudes à appliquer et à diffuser les instructions des services de vulgarisation (Kébédé et al., 1990). En plus, l'éducation formelle détermine positivement le revenu agricole.

L'expérience dans les cultures (NYCROPF): le nombre d'années d'expérience du producteur devrait influencer négativement l'adoption des technologies. L'adoption de nouvelles technologies exige un certain niveau de risque associé à la décision du choix des technologies (Feder, 1982).

La disponibilité de la main d'œuvre (LABOAVAI) : c'est une variable continue qui pourrait influencer positivement l'utilisation de la fumure organique, car, elle nécessite une main d'œuvre subséquente pour le transport et l'épandage. La disponibilité de la main d'œuvre est indispensable pour l'adoption des nouvelles technologies. Le nombre d'actifs agricoles du ménage a été également utilisé par Glèlè et al. (2008) pour montrer sa relation avec l'adoption d'une innovation.

Les connaissances techniques locales (TECHSKIL) : la connaissance d'une technologie est déterminante pour son adoption. Plus les producteurs disposent des connaissances locales, plus ils pourront les adopter ou pas.

L'accès aux crédits (ACCESCRE) : cette variable est déterminante pour le choix d'une stratégie, car, les producteurs ayant un accès facile aux crédits auront tendance à mieux adopter les nouvelles technologies. Car, ils disposeront des moyens financiers nécessaires pour les technologies nouvelles.

L'accès aux ressources (ACCESRES) : cette variable est déterminante pour l'utilisation de la fumure organique dans l'exploitation, car, les producteurs ayant un accès facile aux ressources d'exploitation auront tendance à adopter des stratégies d'adaptation. 
Tableau 1 : Variables utilisées dans le modèle.

\begin{tabular}{|c|c|c|c|c|c|}
\hline Variable & $\begin{array}{l}\text { Description des variables } \\
\text { et de leurs valeurs }\end{array}$ & Moyenne & $\begin{array}{c}\text { Ecart } \\
\text { type }\end{array}$ & Min & Max \\
\hline USMARASO & $\begin{array}{l}\text { Utilisation de la fumure organique } \\
\text { (1 s'il utilise et } 0 \text { sinon) }\end{array}$ & 1,61 & 0,489 & 0 & 1 \\
\hline $\mathrm{AGE}$ & Age de l'enquêté (années) & 45,42 & 12,77 & 25 & 90 \\
\hline GENDER & $\begin{array}{l}\text { Sexe de l'enquêté ( } 1=\text { homme et } \\
0=\text { femme) }\end{array}$ & 1,49 & 0,502 & 0 & 1 \\
\hline MARSTAT & $\begin{array}{l}\text { Situation matrimoniale } \quad(1=\text { marié } \\
\text { et } 0=\text { autres) }\end{array}$ & 1,16 & 0,564 & 0 & 1 \\
\hline LEVEDUC & $\begin{array}{l}\text { Niveau d'éducation }(1=\text { primaire et } \\
0=\text { autres })\end{array}$ & 1,67 & 1,02 & 0 & 1 \\
\hline NYCROPF & $\begin{array}{lll}\text { Nombre } & \text { d'années } & \text { dans } \\
\text { l'agriculture } & & \\
\end{array}$ & 17,80 & 9,87 & 2 & 47 \\
\hline LABOAVAI & $\begin{array}{l}\text { Disponibilité de main d'œuvre } \\
(1=\text { oui, } 0=\text { non })\end{array}$ & 1,69 & 0,463 & 0 & 1 \\
\hline TECHSKIL & $\begin{array}{l}\text { Connaissances techniques locales } \\
(1=\text { oui, } 0=\text { non })\end{array}$ & 1,73 & 0,441 & 0 & 1 \\
\hline ACCESCRE & Accès aux crédits ( $1=$ oui, $0=$ non $)$ & 1,67 & 0,470 & 0 & 1 \\
\hline ACCESRES & $\begin{array}{l}\text { Accès aux ressources } \quad(1=\text { oui, } \\
0=\text { non })\end{array}$ & 1,07 & 0,259 & 0 & 1 \\
\hline LOCLINKS & $\begin{array}{l}\text { Relations sociales dans le village } \\
(1=\text { oui, } 0=\text { non })\end{array}$ & 1,29 & 0,459 & 0 & 1 \\
\hline ACCMARKT & $\begin{array}{l}\text { Accès aux marchés } \quad(1=\text { oui, } \\
0=\text { non })\end{array}$ & 1,24 & 0,430 & 0 & 1 \\
\hline ACCNEWS & $\begin{array}{l}\text { Accès aux informations (1=oui, } \\
0=\text { non })\end{array}$ & 1,090 & 0,287 & 0 & 1 \\
\hline CHILSCHO & $\begin{array}{l}\text { Enfants allant à l'école (1=oui, } \\
0=\text { non })\end{array}$ & 1,49 & 0,502 & 0 & 1 \\
\hline
\end{tabular}

Les relations sociales dans le village (LOCLINKS) : les pratiques des acteurs sociaux sont influencées par les pressions qu'ils subissent de la part des autres acteurs avec qui ils sont en relation. En effet, les acteurs sociaux ne sont pas des acteurs isolés dans un environnement neutre. Ils sont directement ou indirectement en relation avec d'autres acteurs qui les influencent dans leur prise de décision. Ainsi, un acteur peut être amené à rejeter une innovation, non pas parce qu'il n'a pas les ressources nécessaires pour l'adopter, mais parce que son environnement social ne lui permet pas de l'adopter.

L'accès aux marchés (ACCMARKT) : cette variable est déterminante pour le choix stratégique des agriculteurs. L'accès aux marchés permet à la plupart des producteurs d'accéder et d'utiliser les innovations agricoles requises. La plupart des études de cas analysées mettent en relief l'importance capitale des opportunités de marché dans l'incitation à l'investissement dans l'innovation agricole. 
L'accès aux sources d'informations (ACCNEWS) : cette variable est très déterminante pour l'adoption de cette stratégie, car, les agriculteurs plus informés auront tendance à être des novateurs et à adopter des stratégies existantes. Plus les agriculteurs sont informés, plus la probabilité d'adaptation est importante.

L'envoi des enfants à l'école (CHILSCHO) : cette variable est importante dans le modèle, car nous estimons que les agriculteurs ayant des enfants à charge ont besoin des revenus substantiels pour leur éducation. En conséquence, ils auront tendance à adopter des innovations pour améliorer leur revenu. La taille du ménage est souvent mentionnée comme une variable essentielle dans l'adoption de nouvelles technologies. La variable taille du ménage est positivement liée à l'adoption des nouvelles technologies.

\section{RESULTATS}

\section{Caractéristiques socio-économiques des producteurs}

Les producteurs enquêtés dans le département de Kibwezi sont composés à moitié des hommes et des femmes $(50,5 \%$ d'hommes et $49,5 \%$ de femmes). La majorité de ces populations sont mariées $(91,9 \%)$. Le taux de scolarisation est élevé dans cette région, avec près de $87,4 \%$ qui ont été à l'école. L'âge moyen se situe autour de 45 ans et la taille moyenne du ménage est d'environ 6 personnes. Ces populations ont une expérience assez élevée en agriculture d'environ 17 ans et 14 ans pour l'élevage. La majorité de ces paysans ont des parcelles de petites tailles, soit environ 5 ares et un total moyen des parcelles par exploitation de 12 ares (<5 ha). L'agriculture pratiquée est celle de subsistance, soit $73 \%$. Dans leur système de culture, près de $68,5 \%$ utilisent des intrants agricoles tels que les engrais, les pesticides et herbicides et près de $32,4 \%$ utilisent des intrants agroindustriels en élevage. La traction animale reste très utilisée par près de $88,3 \%$ des exploitants. L'accès aux différentes sources d'informations est élevé, avec près de $91 \%$ qui ont accès à l'information (radio, journaux, télévision) et environ 75,7\% ont accès aux marchés. Enfin, seulement $33,3 \%$ ont accès aux services agricoles et environ $32,4 \%$ ont accès aux crédits agricoles.

\section{Effets des changements climatiques sur les} sols

Tous les effets perçus ici par les agriculteurs sont liés entre eux, et directement ou indirectement liés aux changements climatiques. Les effets combinés du caractère orageux et torrentiel des pluies, de l'assèchement et l'induration des sols, favorisent l'érosion hydrique et éolienne qui fait perdre aux sols leur matière organique et les rendant moins fertiles et légers.

De plus, en dehors de la baisse de la fertilité et de l'induration des sols qui sont des phénomènes plus facilement perceptibles par les agriculteurs surtout lors des périodes des semis (induration des sols) et des récoltes (baisse de la fertilité), tous les autres effets sont perçus par une petite minorité (Tableau 2).

\section{Stratégies d'adaptation des agriculteurs face à la baisse de la fertilité des sols}

La variabilité et le changement climatiques peuvent contribuer à la dégradation des terres en exposant les sols non protégés à des phénomènes violents et en compromettant le potentiel des pratiques actuelles de gestion des terres à maintenir la qualité des ressources naturelles, contribuant ainsi au déboisement, à l'érosion des sols, à l'appauvrissement en matières organiques et à d'autres formes de dégradation. C'est dans cette optique que les 
agriculteurs utilisent des stratégies d'adaptation, malgré le fait que le taux d'adoption reste très faible.

Face aux différents risques liés à la variabilité climatique (sécheresse, vents, ennemis des cultures, etc.), les paysans ont développé des stratégies d'adaptation. Parmi ces stratégies, figurent celles relatives aux pratiques agricoles comme la lutte contre la dégradation des terres agricoles, la gestion de la fertilité des sols, les techniques de gestion de l'eau, la diversification des cultures, etc. Dans le département de Kibwezi, il existe une très faible adoption des stratégies d'adaptation par les agriculteurs face aux effets des changements climatiques sur les sols; ce qui traduit les difficultés d'adaptation de ces agriculteurs à cause de la pauvreté, ou du manque de collaboration avec les structures d'encadrement agricole.

Parmi ces stratégies, la plus utilisée reste l'utilisation des terrasses (87\%) car dans cette région, l'érosion hydrique est très accélérée à cause du relief parfois en pente, qui cause d'énormes pertes de matières fertilisantes aux sols. Ces terrasses constituent des obstacles qui ralentissent la vitesse de ruissellement, permettent la sédimentation et le dépôt des particules (sables, terre fine, matière organique) à l'amont de la terrasse, et augmentent l'infiltration des eaux ruisselantes.

La fumure organique, utilisée par près de $39 \%$ des agriculteurs face aux effets des changements climatiques sur les sols, pourrait s'expliquer par une prise de conscience collective des agriculteurs par rapport au lien qui existe entre tous les autres effets sur les sols et la baisse de la fertilité des sols. Aussi, près de 80\% des exploitants possèdent des ruminants pour la réalisation des opérations de labour et de la fertilisation (apport du fumier) des champs. Il ressort de ce résultat que l'utilisation de la fumure organique est peu répandue chez les producteurs d'une manière globale.
En outre, face à la baisse de la fertilité, la pratique de l'agroforesterie, chez les agriculteurs est très limitée $(5 \%)$, notamment chez les agriculteurs allochtones.

La plupart des producteurs sont persuadés de l'importance de l'agroforesterie, mais ils opèrent d'autres choix. La raison évoquée est la tenure du foncier. Selon les producteurs allochtones, ils n'ont pas le droit de planter des arbres sur des parcelles louées, car l'arbre sur une parcelle est un indicateur d'appropriation des terres. Dans de telles conditions, le renouvellement du stock organique par les feuilles des ligneux et par l'activité racinaire n'est pas assuré. L'usage de l'irrigation est une des stratégies possibles, mais, il est à constater que très peu d'agriculteurs l'adoptent (moins de 5\%). En effet, la maîtrise de l'eau passe par le passage d'une agriculture pluviale à une autre irriguée, en favorisant les investissements dans des petits ouvrages hydro-agricoles à rentabilité économique assurée et en réorientant la politique sectorielle vers la gestion intégrée des ressources en eau. Pour augmenter la production agricole dans une situation de stress hydrique fréquent et de forte demande de produits agricoles, il convient d'accroître la productivité de l'eau et des intrants.

Enfin, l'usage des engrais face à la baisse de la fertilité est une stratégie d'adaptation, mais, une proportion très marginale l'adopte. Cela peut se justifier par la pauvreté des agriculteurs et les coûts d'accès aux engrais chimiques élevés. D'autres stratégies existent, mais restent très faiblement utilisées par les agriculteurs probablement à cause de certains facteurs socio-économiques influençant l'adoption (Tableau 3). 
Déterminants d'utilisation de la fumure organique face à la baisse de la fertilité

Nous rappelons que la variable expliquée est représentée par l'utilisation de la fumure organique pour s'adapter à la baisse de la fertilité induite par les changements climatiques dans la région semi-aride de Kibwezi. Les résultats d'estimation du modèle Logit sont consignés dans le Tableau 4.

Les résultats mettent en évidence une corrélation entre les facteurs socioéconomiques et l'utilisation de la fumure organique. L'estimation du modèle Logit a permis de constater que les variables socioéconomiques telles que le statut matrimonial, les connaissances techniques et locales, l'accès aux ressources, les relations sociales dans le village ont une influence significative sur la probabilité d'utilisation de la fumure organique face à la baisse de la fertilité due aux changements climatiques au seuil de 5\% et le niveau d'éducation des chefs de ménage au seuil de $10 \%$. Parmi ces variables, certaines ont des effets positifs sur la probabilité d'utilisation, notamment, le statut matrimonial et les relations sociales dans le village, tandis que d'autres affectent négativement le choix de la stratégie, notamment, le niveau d'éducation, les connaissances techniques et l'accès aux ressources et autres.

Tableau 2: Effets directs ou indirects des changements climatiques sur les sols.

\begin{tabular}{lcc}
\hline Effets perçus par les agriculteurs & Fréquence & Pourcentage (\%) \\
\hline Baisse de la fertilité des sols & 93 & 83,8 \\
Induration de la surface des sols & 64 & 57,7 \\
Modification de la couleur des sols & 12 & 10,8 \\
Accroissement de l'érosion des sols & 10 & 9,0 \\
Sols légers & 3 & 2,7 \\
Assèchement ou compaction des sols & 2 & 1,8 \\
\hline
\end{tabular}

Tableau 3 : Stratégies d'adaptation des agriculteurs.

\begin{tabular}{lcc}
\hline Stratégies d'adaptation adoptées & Fréquence & Pourcentage (\%) \\
\hline Terrasses & 97 & 87,4 \\
Fumure organique & 43 & 38,7 \\
Plantation d'arbres & 6 & 5,4 \\
Irrigation des sols & 5 & 4,5 \\
Usage des engrais & 4 & 3,5 \\
Usage de la charrue pour casser les sols indurés & 1 & 0,9 \\
\hline
\end{tabular}


Tableau 4 : Modèle Logit d'utilisation de la fumure organique face à la baisse de la fertilité des sols.

\begin{tabular}{|c|c|c|c|c|}
\hline Variable & Estimation & Ecart type & Wald & Significativité \\
\hline Constante & $-1,206$ & 2,239 & 0,290 & 0,590 \\
\hline AGE & 0,036 & 0,031 & 1,400 & 0,237 \\
\hline GENDER & $-0,676$ & 0,516 & 1,716 & 0,190 \\
\hline MARSTAT & 1,505 & 0,670 & 5,044 & $0,025^{* *}$ \\
\hline LEVEDUC & $-0,581$ & 0,320 & 3,284 & $0,070 *$ \\
\hline NYCROPF & $-0,043$ & 0,035 & 1,553 & 0,213 \\
\hline EXTASSIS & 0,841 & 0,805 & 1,092 & 0,296 \\
\hline LABOAVAI & 0,787 & 0,687 & 1,311 & 0,252 \\
\hline TECHSKIL & $-1,674$ & 0,812 & 4,245 & $0,039 * *$ \\
\hline ACCESCRE & 0,351 & 0,599 & 0,344 & 0,558 \\
\hline ACCESRES & $-2,731$ & 1,220 & 5,005 & $0,025^{* *}$ \\
\hline LOCLINKS & 1,410 & 0,623 & 5,121 & $0,024 * *$ \\
\hline ACCMARKT & $-0,751$ & 0,683 & 1,210 & 0,271 \\
\hline ACCNEWS & 1,564 & 1,164 & 1,806 & 0,179 \\
\hline CHILSCHO & $-0,924$ & 0,604 & 2,341 & 0,126 \\
\hline Nombres d'observations & & \multicolumn{3}{|c|}{111} \\
\hline Log-vraisemblance & & \multicolumn{3}{|c|}{$-148,199$} \\
\hline Pseudo R2 & & \multicolumn{3}{|c|}{0,266} \\
\hline Khi 2 & & \multicolumn{3}{|c|}{39,38} \\
\hline Prob & & \multicolumn{3}{|c|}{0,000} \\
\hline
\end{tabular}

Les niveaux de significativité sont respectivement : $1 \%(* * *), 5 \%(* *)$ et $10 \%(*)$.

\section{DISCUSSION}

Les changements climatiques entrânent une détérioration de l'environnement (Berestovoy et Fluet, 2005). Ainsi, les paysans perçoivent les changements des précipitations à travers ses effets directs sur les sols et le couvert végétal. Au Burkina Faso, environ $36 \%$ des exploitants en zone sahélienne explique la faiblesse relative de la perception de la baisse des précipitations dans cette zone (Ouédraogo et al., 2010).

Les perceptions paysannes des changements du climat corroborent les observations météorologiques et la littérature sur la crise climatique en Afrique subsaharienne. Par exemple, la baisse des précipitations perçue par les paysans a été rapportée par plusieurs auteurs en des termes différents : déficit pluviométrique persistant ; assèchement du Sahel (GIEC, 2007). La perception des changements des précipitations par ses effets directs sur les sols et le couvert végétal peut se comprendre à travers les liens qui existent entre, d'une part, la dégradation des terres et les changements climatiques et, d'autre part, la dégradation des forêts et les changements climatiques. L'appauvrissement de la flore est le premier constat d'une sécheresse prolongée qui a affecté tout le Sahel (Folefack et al., 2012). La sécheresse figure parmi les variations climatiques au Sahel qui jouent un rôle très important dans le processus de dégradation des terres. L'aridité expose les sols aux processus d'érosion éolienne et hydrique entrânant le départ de la partie arable et 
fertile des sols, ralentit les processus biologiques au niveau des sols et entraîne notablement une baisse de leur productivité. Certains changements dans les paysages reflètent le changement climatique global (Salé, 2010).

En outre, parmi les stratégies d'adaptation aux changements chimiques figurent en bonne place, l'utilisation de la fumure organique dans les exploitations. Elle consiste en un apport de fumier et/ou de compost. Certains paysans parquent les animaux dans leurs champs pour profiter des déjections. Les taux d'adoption de la fumure organique reste faible (seulement $38 \%$ ). Ce taux est proche de celui de la zone sahélienne trouvé par Ouédraogo et al. (2010) et qui variait inversement avec le gradient des précipitations (39\% en zone sahélienne, $28 \%$ en zone soudano-sahélienne et $13 \%$ en zone soudanienne). Les sécheresses et la baisse des pluies ont engendré une perte du couvert végétal, entraînant une baisse de la fertilité des sols. La dégradation des sols est plus ressentie par les paysans dans un contexte de crise climatique. C'est pourquoi la fumure organique, qui est utilisée en réponse à la baisse de la fertilité, est considérée par certains paysans comme une stratégie d'adaptation au changement des précipitations.

Par ailleurs, l'étude a permis de mieux comprendre les facteurs socio-économiques qui influencent l'adoption de la fumure organique pour faire face à la baisse de la fertilité dans un contexte de changements climatiques dans la région de Kibwezi. Une analyse de la sensibilité de la probabilité d'adoption par rapport aux variables explicatives montre que certains variables socio économiques ont des effets marginaux les plus forts, en plus, certaines variables affectent négativement la propension à l'adoption de la stratégie.
Le statut matrimonial (MARSTAT) est corrélé positivement à la probabilité des agriculteurs d'utiliser cette stratégie d'adaptation au seuil de 5\%. Les agriculteurs mariés sont ceux qui possèdent des enfants leur servant de main d'œuvre pour l'entretien des animaux, la collecte et l'épandage de la fumure pour améliorer la fertilité de leur sol et accroître leur revenu agricole et satisfaire les besoins de l'exploitation et de la famille. Le statut matrimonial détermine les besoins et les dépenses du producteur, et conditionne ses décisions d'améliorer sa productivité agricole.

Le niveau d'éducation (LEVEDUC) quant à lui influence négativement le choix de cette stratégie au seuil de $10 \%$. Plus le niveau des agriculteurs est élevé, moins ils auront tendance à adopter cette stratégie d'adaptation face à la baisse de la fertilité. Dans cette zone, les producteurs ayant un niveau d'éducation élevé sont généralement des fonctionnaires qui travaillent ailleurs, et donc n'ont pas assez de temps pour se consacrer à une technique contraignante, et ont accès à plusieurs sources d'informations qui leur permettent d'utiliser d'autres stratégies d'adaptation leur permettant d'améliorer leur production agricole. L'éducation est un facteur affectant l'adoption et l'application des innovations technologiques en milieu rural (Azontondé, 2004).

Les connaissances techniques locales (TECHSKIL) ont eu des effets significatifs sur la probabilité du choix de la stratégie au seuil de $5 \%$, Cependant, il est à relever qu'avec le signe négatif du coefficient, on constate que plus les agriculteurs disposent des techniques locales, ils auront tendance à moins adopter cette stratégie d'adaptation. Cela parce que ces derniers connaissent d'autres stratégies moins contraignantes pour faire face aux problèmes liés aux sols. 
L'accès aux ressources (ACCESRES) (terre, semences) est une variable qui a eu des effets significatifs au seuil de 5\%. Cependant, le signe négatif observé fait croire que les agriculteurs ayant plus de ressources dans les exploitations auront tendance à moins adopter cette stratégie. Car, les grands propriétaires terriens ne sont pas prêts à utiliser la fumure organique à cause des quantités importantes exigées, et surtout que la disponibilité de cette fumure pose problème du fait de l'abandon progressif de l'élevage des bovins suite à la sécheresse due aux changements climatiques. Le même résultat a été obtenu par Ouédraogo et al. (2010) car, la variable tenure affecte négativement l'adoption de la fumure, ce qui est contraire à l'hypothèse communément admise selon laquelle, la crainte de se voir déposséder d'une terre empruntée une fois valorisée limiterait les investissements sur cette terre. Les terres facilement accessibles étant les moins productives, l'emprunteur est obligé d'améliorer la qualité de ces terres à travers l'utilisation de la fumure.

En plus, on observe des effets significatifs $(5 \%)$ de la variable relation sociale dans le village (LOCLINKS) sur la probabilité du choix de la stratégie. Cette variable influence positivement l'adoption, ce qui stipule que les agriculteurs ayant des liens sociaux solides dans le village auront tendance à plus adopter cette stratégie d'adaptation. Cela conforte notre assomption que les individus ne vivent pas en vase clos, ils sont influencés par leur entourage. Les pratiques des acteurs sociaux sont influencées par les pressions qu'ils subissent de la part des autres acteurs avec qui ils sont en relation. En effet, les acteurs sociaux ne sont pas des acteurs isolés dans un environnement neutre. Ils sont directement ou indirectement en relation avec d'autres acteurs qui les influencent dans leur prise de décision.
Notons enfin que d'autres variables à priori pertinentes, présentes dans le modèle, n'ont aucun effet significatif sur le choix des stratégies par les agriculteurs, bien que ces variables influencent le choix de la stratégie d'adaptation. Il s'agit notamment de l'âge du producteur, le sexe, l'assistance extérieure dans l'exploitation, l'expérience dans l'agriculture, la disponibilité de main d'œuvre, l'accès aux crédits, aux marchés et aux informations et l'envoi des enfants à l'école.

\section{Conclusion}

La présente étude a permis d'identifier les facteurs socio-économiques qui influencent l'utilisation de la fumure organique face à la baisse de la fertilité des sols, dans un contexte de changements climatiques dans la région semi-aride de Kibwezi. Le modèle Logit a été mobilisé pour isoler ces variables à partir des données collectées auprès des producteurs de la zone semi-aride de Kibwezi. Les paysans perçoivent clairement les changements climatiques opérés au niveau des précipitations et de la hausse des températures. Les effets des changements climatiques sur les sols restent majoritairement, la baisse de la fertilité, l'induration et la modification de la couleur des sols. Ces perceptions sont plus importantes dans les zones les plus vulnérables. Face à ces effets, les producteurs adoptent des stratégies d'adaptation, dont les plus marquantes sont les terrasses et l'utilisation de la fumure organique face la baisse de la fertilité des sols.

A l'aide du modèle Logit, on constate qu'une corrélation existe entre les facteurs socio-économiques et l'utilisation de la stratégie. On constate que les variables socioéconomiques telles que le statut matrimonial, les connaissances techniques et locales, 
l'accès aux ressources, les relations sociales dans le village ont une influence significative sur la probabilité d'utilisation de la fumure organique face à la baisse de la fertilité due aux changements climatiques au seuil de $5 \%$ et le niveau d'éducation des chefs de ménage au seuil de $10 \%$. Parmi ces variables, certaines ont des effets positifs sur la probabilité d'utilisation, notamment, le statut matrimonial et les relations sociales dans le village, tandis que, d'autres affectent négativement le choix de la stratégie, notamment, le niveau d'éducation, les connaissances techniques et l'accès aux ressources.

Notons enfin que d'autres variables, à priori pertinentes, présentes dans le modèle, n'ont aucun effet significatif sur le choix des stratégies par les agriculteurs, bien que ces variables influencent le choix de la stratégie d'adaptation. Il s'agit notamment de l'âge du producteur, le sexe, l'assistance extérieure dans l'exploitation, l'expérience dans l'agriculture, la disponibilité de main d'œuvre, l'accès aux crédits, aux marchés et aux informations et l'envoi des enfants à l'école. Ainsi, afin d'augmenter la probabilité d'adopter la fumure organique face à la baisse de la fertilité, des recommandations suivantes peuvent être formulées :

- Renforcer la sensibilisation des agriculteurs par rapport à l'importance de la fumure organique pour la fertilité des sols en passant des messages à travers les différentes organisations sociales dans les villages (GIC, tontines, associations);

- Inciter et appuyer ces agriculteurs à militer dans des groupements associatifs tels que les GIC ou les tontines. Car, les relations sociales dans le village sont également importantes, et dans ce cas, le gouvernement, à travers les services techniques compétents (agriculture, élevage), encourage ces initiatives ;
- Améliorer l'accès des agriculteurs aux structures d'encadrement agricole ;

- résoudre le problème foncier en allouant des portions de terre aux nouveaux immigrants dans les différents villages ;

- Encourager la fabrication et l'usage du compost à la place de la fumure dont la disponibilité est limité à cause de l'abandon progressif de l'élevage des bovins.

\section{REFERENCES}

Adesina AA, Mbila D, Nkamleu GB, Endamana D. 2000. Econometric analysis of the determinants of adoption of alley farming by farmers in the forest zone of southwest Cameroon. Agriculture, Ecosystems \& Environment, 80: 255-265.

Azontondé RPE. 2004. Impact économique de l'adoption des pratiques de la gestion intégrée de la fertilité des sols (GIFS) au Sud-Bénin : cas d'Ahohoué (Commune de Klouékanmè) et de Banigbé (Commune d'Ifangni).Thèse pour l'obtention du Diplôme d'Ingénieur Agronome ; 126 p.

Berg A, Noblet-Ducoudré N, Sultan B, Lengaigne M, Guimberteau M. 2012. Projections of climate change impacts on potential crop productivity over tropical regions. Agricultural and Forest Meteorology, doi:10.1016/j.agrformet. 2011.12.003.

Berestovoy P, Fluet MJ. 2005. De Lachine au Sahel : perceptions des changements climatiques. FrancVert 2005, $2: 7$ p.

Challinor A, Wheeler T, Garforth C, Craufurd P, Kassam A. 2007. Assessing the vulnerability of food crop systems in Africa to climate change. Climatic Change, 83: 381-399.

Chow GC. 1983. "Econometrics". Mc GrawHill, Inc., 3rd Printing 1987, Singapore. 
Cramer JS. 1991. The Logit Model for Economists: London and New York: Edward Arnold.

FAO. 2007. Interaction entre agriculture et forêt. 20ème session, Comité de l'agriculture, Rome, 25-28 avril 20074 p.

Feder G. 1982. On export and economic growth. Journal of Development Economics, 12: 59-73.

Folefack DP, Sale A, Wakponou A. 2012. Facteurs affectant l'utilisation de la fumure organique dans les exploitations agricoles en zone sahélienne du Cameroun. Afrique Science: Revue Internationale des Sciences et Technologie, 8(2): 22-33.

Glele EKA, Adekambi AS, Agli CK, Tamegnon BA, Adegbola PY. 2008. Impact socio-économique de l'adoption des variétés améliorées de manioc au Bénin. 51 p.

GIEC, 2007. Bilan 2007 des changements climatiques: impacts, adaptation et vulnérabilité. Editions GIEC: Paris, France; 39 p.

Greene WH. 1991. Econometric Analysis. Englewood Cliffs, NJ. Prentice Hall.

Hansen J, Challinor A, Ines A, Wheeler T, Moron V. 2006. Translating climate forecasts into agricultural terms: advances and challenges. Climate Research, 33(1) : 27.

Hurlin C. 2003. Econométrie des variables qualitatives. Cours de maîtrise d'économétrie, France Université d'Orléans, 59 p.

Kebede Y, Gunjal K, Coffin G. 1990. Adoption of new technologies in ethiopian agriculture: the case of teguletbulga district, Shoa Province. Agric. Econ., 4 : 27-43.

Kieken H. 2007. Changements climatiques: prévenir, s'adapter, changer les politiques de développement? Grain de Sel, 38 : 3233.

Oram PA. 1989. Sensitivity of agricultural production to climatic change, an update. In Climate and Food Security. IRRI: Manila, The Philippines; 25-44.

Ouédraogo M, Dembélé Y, Somé L. 2010. Perceptions et stratégies d'adaptation aux changements des précipitations : cas des paysans du Burkina Faso. Science et Changements Planétaires / Sécheresse, 21(2): 87-96.

Long JS. 1997. Regression Models for Categorical and Limited Dependent Variables (Advanced Quantitative Techniques in the Social Sciences). Sage Publications.

Madalla GS. 1985. Limited Dependent and Qualitative Variables in Econometrics. Cambridge University Press: New York.

McFadden D. 1973. Conditional Logit analysis of qualitative choice behavior. In Frontiers in Econometrics, Zarembemka P (ed). Academic Press: New York.

MOA. 2009. Activities annual report. Kibwezi district, Kenya. 25 p.

Nacro S, Ouedraogo S, Traore K, Sankara E, Kabore C, Ouattara B. 2010. Effets comparés des pratiques paysannes et des bonnes pratiques agricoles de gestion de la fertilité des sols sur les propriétés des sols et les rendements des cultures dans la zone sud soudanienne du Burkina Faso. Int. J. Biol. Chem. Sci., 4(4): 1044-1055.

Neupane RP, Sharma KR, Thapa GB. 2002. Adoption of agroforestry in the hills of Nepal: A logistic regression analysis. Agricultural Systems, 72: 177-196.

Nkamleu GB, Kielland A. 2006. Modeling farmers' decisions on child labor and schooling in the cocoa sector: a multinomial Logit analysis in Côte 
d'Ivoire. Agricultural Economics, 35: 319-333.

Nkamleu GB, Adesina AA. 2000. Determinant of chemical input use in peri-urban lowland systems : bivariate probit analysis in Cameroon. Agr. Systems, 62: 1-11.

Nkamleu GB, Coulibaly O. 2000. Les déterminants du choix des méthodes de lutte contre les pestes dans les plantations de cacao et café du sud-Cameroun. Revue Economie Rurale, 259: 75-85.

PNUD. 2004. Reducing disaster risk: a challenge for development. UNDP global report (ed. M. Pelling).

Salé A. 2010. Identification of the factors influencing farmers' adoption of climate change agricultural adaptation strategies and consequent agricultural natural resources management policies in the arid areas of Africa: case of Kibwézi district (Kenya). Technical report (2009/2010 START/IDRC climate change fellowship). $32 \mathrm{p}$.

Sultan B. 2011. L'étude des variations et du changement climatique en Afrique de l'Ouest et ses retombées sociétales. Habilitation à diriger des recherches, Université Pierre et Marie Curie, 137 p.

Varian HR. 2006. Analyse Microéconomique (6e édn). De Boeck : Bruxelles ; 824 p.

Zombré NP. 2003. Les sols très dégradés (Zipela) au centre nord du Burkina Faso : Dynamique, caractéristiques morphobiopédologiques et impacts des techniques de restaurations. Thèse de doctorat. Université de Ouagadougou, Burkina Faso, 146 p. 